\title{
LA-UR-19-29821
}

Approved for public release; distribution is unlimited.

Title:

Author(s):

Issued:
Science Requirements for the MDL

Harwell, Megan Louise

Plesko, Catherine Suzanne

Becker, Stephen Allan

Biwer, Christopher Michael

Margolin, Len G.

Boslough, Mark B.

Intended for: $\quad$ Supplemental information on scientific data requested for Mission Design Lab at Goddard during week of August 26, 2019 
Disclaimer:

Los Alamos National Laboratory, an affirmative action/equal opportunity employer, is operated by Triad National Security, LLC for the National Nuclear Security Administration of U.S. Department of Energy under contract 89233218CNA000001. By approving this article, the publisher recognizes that the U.S. Government retains nonexclusive, royalty-free license to publish or reproduce the published form of this contribution, or to allow others to do so, for U.S. Government purposes. Los Alamos National Laboratory requests that the publisher identify this article as work performed under the auspices of the U.S. Department of Energy. Los Alamos National Laboratory strongly supports academic freedom and a researcher's right to publish; as an institution, however, the Laboratory does not endorse the viewpoint of a publication or guarantee its technical correctness. 


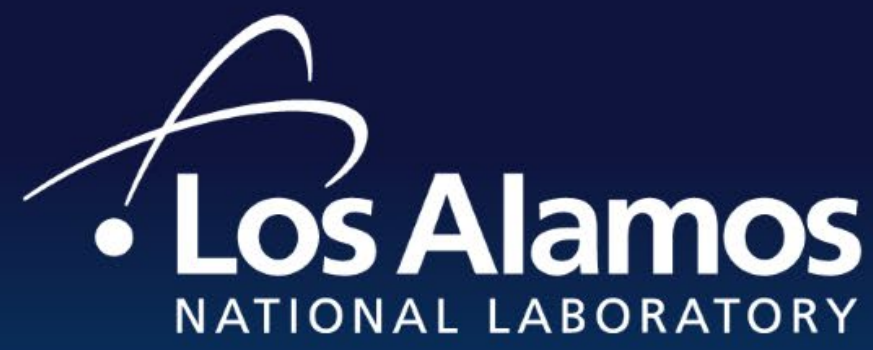

EST. 1943

\section{Science Requirements for the MDL}

Megan Harwell, Cathy Plesko, Steve Becker, Chris Biwer, Len Margolin, Mark Boslough

\section{$9 / 23 / 2019$}




\section{Hellocentric Orbit state}

- Observed during flyby through visual and IR cameras

- Measured to reasonably high fidelity during flyby with combination of Visual and IR cameras and Deep Space Network (DSN), which provides spacecraft telemetry for NASA spacecraft, tracking spacecraft closely

- Improve calculated probability of Earth impact, the angle and the location of impact

- Answers "Will it hit us?" to high certainty

- Uncertainty in path of NEO as calculated from ground based observations

- Images from nav cams on flyby approach are useful for slight adjustments in trajectory of spacecraft. The NEO position data along with DSN tracking of the spacecraft reduces uncertainty in position of NEO

Earth,

DSN

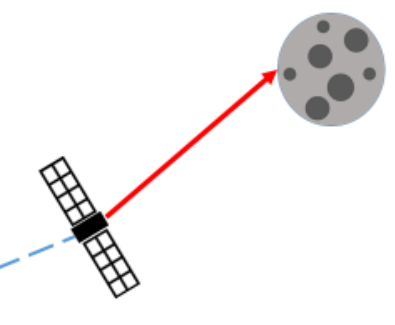




\section{Mass cont.}

\section{What is observed cont:}

- Flyby with vis and IR: observe light spectra from surface of asteroid to obtain mineral composition

- As sunlight hits surface of NEO, minerals on surface absorb some wavelengths of light and reflect others, creating a unique reflectance spectrum read by the vis camera.

- In the IR spectrum, asteroid surface absorbs heat during "day" and radiates it during "night" as it cools. The IR camera picks up on the radiated light, collecting the emission spectra of the surface

- Example: spectrum from burning elements with Bunsen burner in high school chemistry produces flames of different colors (Copper produced green flame, cobalt, blue)

- Compare collected spectra from asteroid to database of mineral spectra to identify surface composition of asteroid.

- Minerals have a known crystalline density (no porosity)

- Multiply crystalline density with approximate volume to obtain upper bound on mass.

However, missions that interact with the object measure about $50 \%$ of estimated mass
- Los Alamos NATIONAL LABORATORY

EST.1943

Atomic Hydrogen emission spectrum (top) and absorption spectrum (bottom)

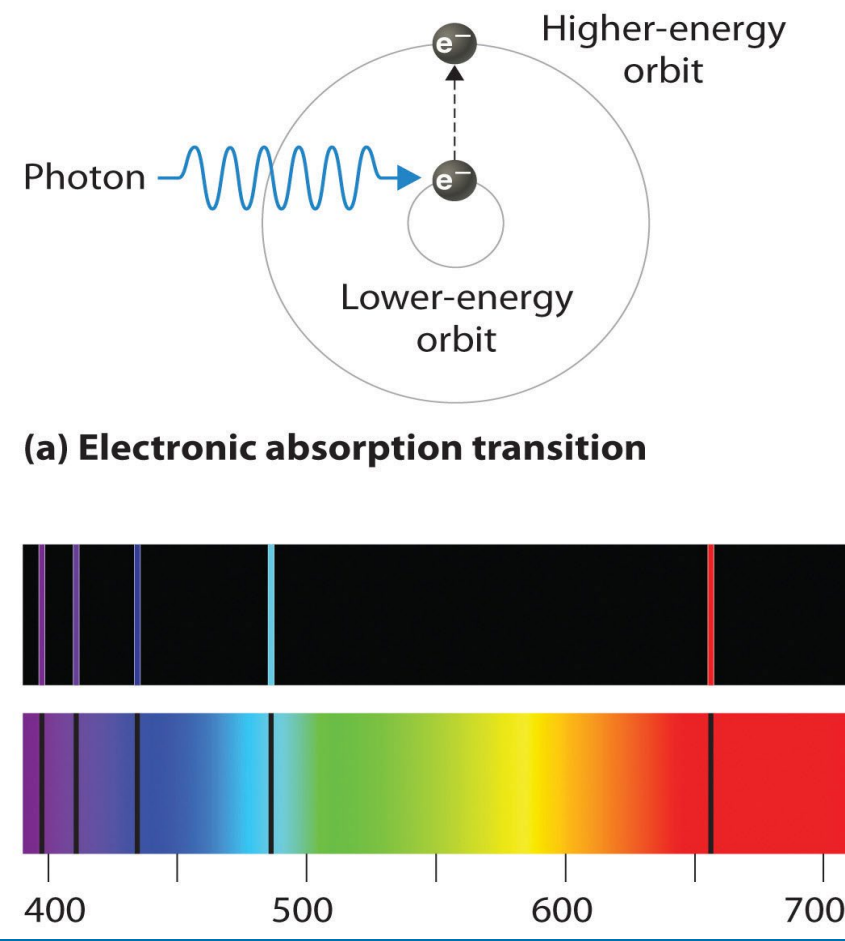

Image credit: Libretexts.org, ch. 7.3: The Atomic Spectrum of Hydrogen

(https://chem.libretexts.org/Courses/Solano_Community_C ollege/Chem_160/Chapter_07\%3A_Atomic_Structure_and Periodicity/7.03_The_Atomic_Spectrum_of_Hydrogen) 


\section{Binarity}

- Observed through visible and IR on flyby and in rendezvous

- IR and vis observations of binary informs deflection mission: If we successfully deflect primary asteroid and miss binary, binary could still be on earth intercept orbit and cause potentially catastrophic damage.

- Interested in binary objects greater $>=20 \mathrm{~m}$ in diameter, based on Chelyabinsk impactor

- Detected when resolved at less than a pixel

- Hill radius/sphere of influence gives upper limit of radius of orbit from primary

$-r_{h}=a(1-e)\left(\frac{m_{\text {asteroid }}}{3 M_{\text {solar }}}\right)^{1 / 3} \sim 8 \mathrm{~km}$

- Radius from asteroid at which gravitational pull from

- sun and asteroid provides equal gravitational force

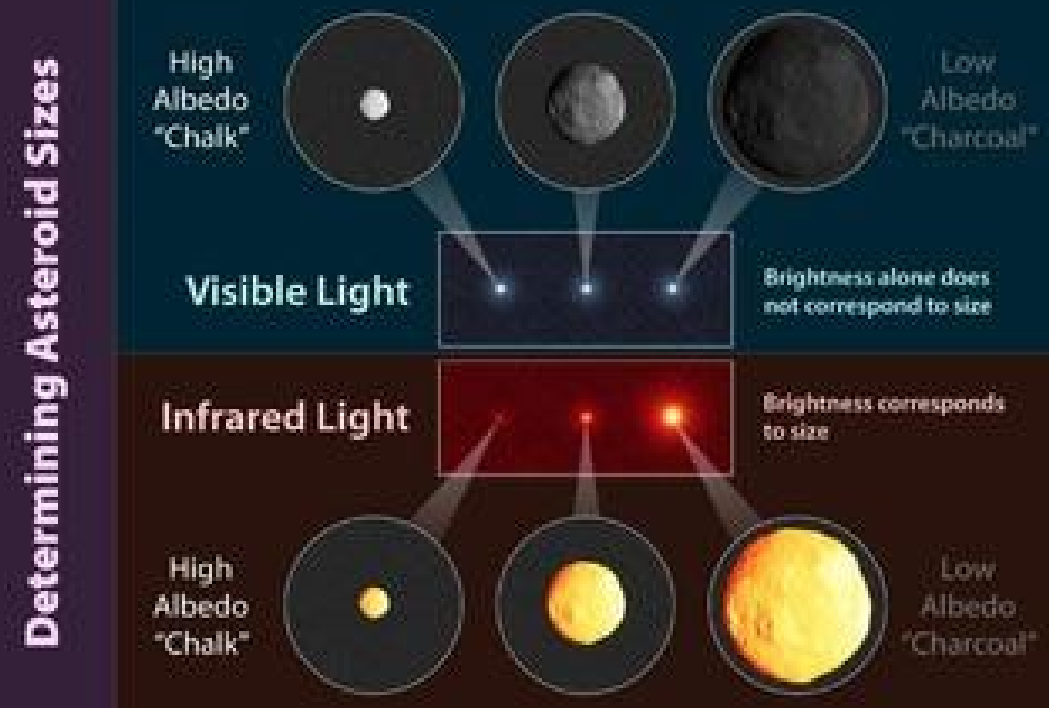




\section{Body bounding sphere and Best fit tos Alamos triaxial}

- Possibly observed in flyby, definitely in rendezvous in both vis and IR

- Images in both vis and IR across more surfaces of body will improve asteroid shape model over time

- From flyby images, we may be able to approximate smallest sphere that encompasses entire asteroid. This gives a rough shape model for simplified or preliminary numerical models

- Further images, likely during rendezvous, will give us better bounds on the axis, allowing for a triaxial ellipsoid shape model. These will be slightly more advanced, but still preliminary shape models. 
- Possibly observed in flyby, definitely in rendezvous in both vis and IR

- Combination of shape model, mass, and surface images inform where effectiveness of deflection effort is maximized

- Strongly positive or negative topographic features around traget region affect energy deposition and momentum transfer to object

- Improve safety and effectiveness with numerical models and accurate shape models 


\section{Surface roughness within 2-sigma}

\section{targeting error around surface target point}

- Observed in rendezvous in both vis and IR and may be able to gather small amount of data during flyby

- Roughness of surface within the circular error probable of target on NEO's surface

- $\quad$ "For nuclear standoff burst the standoff distance will generally be large enough that enough surface area is being integrated over that surface details are relatively less important. However, kinetic impactor performance may depend more strongly on local features of the impact point on the asteroid's surface." - From PD_NEO_Characterization_Matrix.xIsx

- In Kinetic Impactor deflection scenario, energy is deposited in small area on the surface of the NEO. The local topography, or surface features within the targeting error for the deflection attempt, will more greatly affect the efficiency of momentum transfer to the center of mass of the NEO.

- For example, a large boulder near the target could be lofted at very high speed, leaching momentum intended for deflection

- During a nuclear standoff deflection scenario, energy is deposited onto surface area proportional to height of burst. Due to larger surface area for energy deposition, individual topographic features within 2 sigma of target on surface of NEO has less of an effect on effectiveness of momentum transfer 
- Possibly observed in flyby, definitely in rendezvous in both vis and IR

- May be able to collect data during flyby depending on solar phase angle at time of approach, flyby speed and geometry relative NEO, and asteroid's shape, size, and rotational period

- Because data is obtained through processing of images taken over time, data collection best during rendezvous

- From rotational state, we gather for asteroid's numerical material model and equation of state

- Bulk cohesion, compressive strength, tensile strength, shear strength

- Rotational state will affect targeting 


\section{Strengths from rotational state}

- Data gathered during rendezvous with Vis, IR, and neutron spectrometer instruments

- Value will be estimated from composition gathered by spectra, bulk porosity estimated from gravitational pull experienced by flyby during close approaches, and near-surface density as measured by the neutrons spectrometer

- Strength parameters allow us to find the most appropriate material model and equation of state for the asteroid in hydrocode models.

- Bulk cohesion:

- Lower bound on bulk cohesion obtained through cohesive strength required to keep asteroid solid at rate of rotation (Doesn't angle of repose play into this as well?)

- F_cent $<=$ F_cohesive... What is actual formula? 
- Compressive strength, or resistance of the material to breaking under compression

- Will hitting it too hard cause disruption?

- Tensile Strength: Resistance of a material to breaking under tension

- Will a rarefaction destroy the asteroid?

- Shear strength: Resistance of a material to failing under a shearing, or sliding, load

- Weak lower bound informed by angle of repose of surface regolith and topographic highs from images 


\section{Bulk porosity}

- Data gathered during rendezvous through DSN tracking of spacecraft during post-rendezvous close proximity operations

- $m_{\text {measured }}=\phi m_{\text {estimated }}$

- Quality gravity measurements allow for the direct measurement of the celestial body's mass.

- $\quad m_{\text {estimated }}$, the prior assumption for mass was based on crystalline density of the bulk mineral composition, giving a strong upper bound on mass. As stated earlier, measured mass is typically $\sim 50 \%$ of estimated mass. Assuming the same material composition, the difference in mass can be attributed to porosity and mass variations, $\phi$

- Large mass anomalies, including caves and regions of much higher density, detected in the rendezvous phase can be excluded from the estimation for bulk density to give a more realistic estimation.

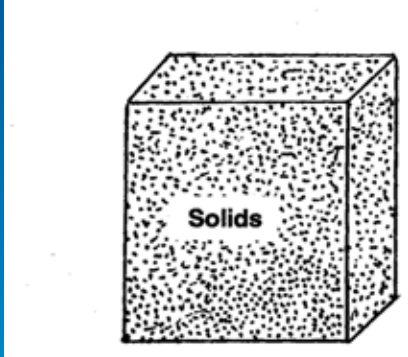

Particle Density

$100 \%$ solid Weight $=2.66 \mathrm{~g}$ Volume $=1 \mathrm{~cm}^{3}$
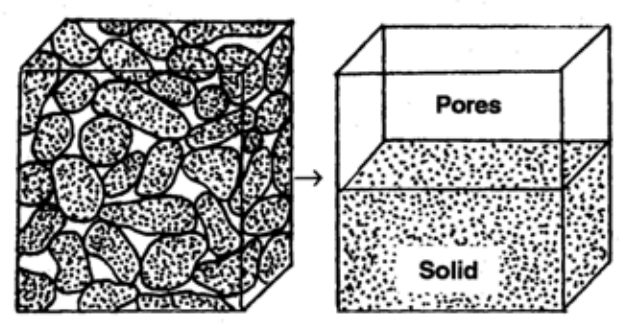

Bulk Density

$50 \%$ solid, $50 \%$ pore space Weight $=1.33 \mathrm{~g}$

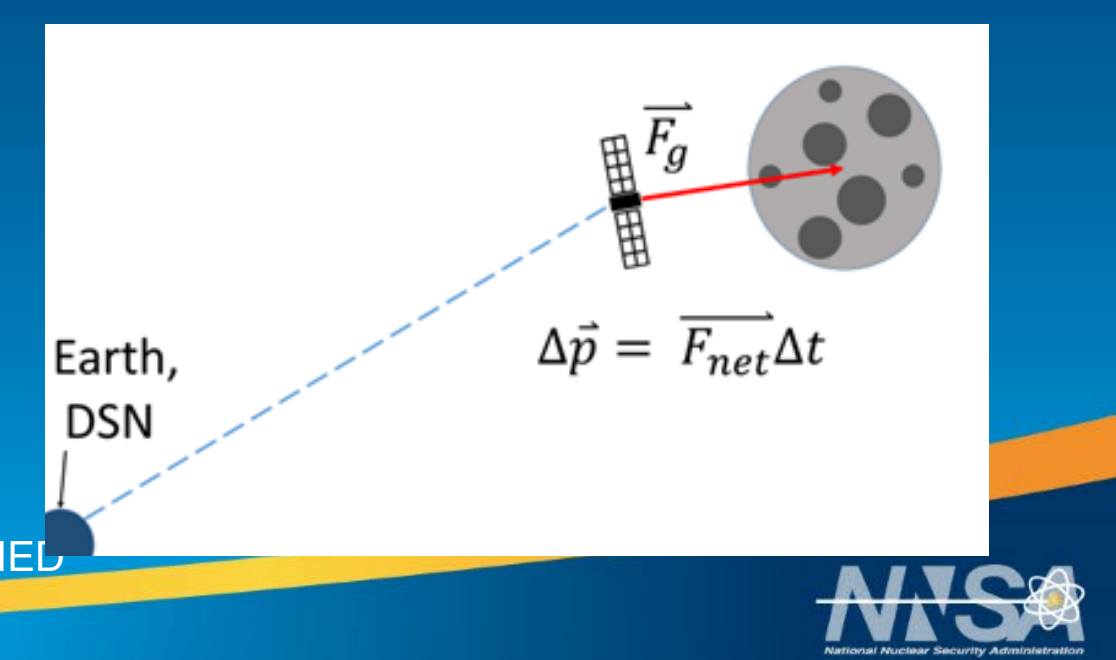




\section{Gravity field (mascons)}

Data gathered during rendezvous through visual and IR camera ând DSN

- Can be solved for from data gathered during rendezvous through DSN tracking of spacecraft during post-rendezvous close proximity operations and optical nav tracking of asteroid

- Mass Concentrations in asteroid may indicate obscured boulders or a mass of iron

- Likely fourth order is Highest spherical harmonic order possible to solve for through these methods

- If greater resolution needed, other options include gradiometer and surfacepenetrating radar (tomography)

- Mascons may reduce the effectiveness of the momentum exchange during deflection effort.

\section{Shock wave}

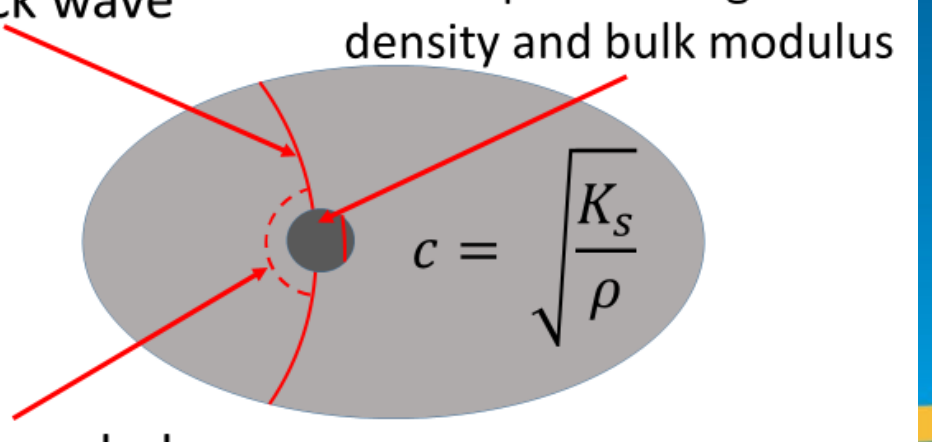

Shock speed changes with

Higher density/concentrations of greater than surrounding mass change how the shock wave moves through the asteroid and may cause rebounding waves that interfere with the wave front.

UNCLASSIFIED 
- Possibly observed in flyby, observed during rendezvous with vis and IR cameras as well as neutron spectrometer

- Rough estimate for composition observed through spectra from surface of asteroid, refined through gamma ray and neutron spectroscopy to obtain more accurate components of composition

- A more thorough understanding of composition means we can apply an equation of state that will be the most representative of the bulk composition of the asteroid, leading to more accurate numerical models

- Combination of fractions of EOSes along with their corresponding opacity values give the models more fidelity regarding comes to energy deposition onto NEO 
- Data gathered by neutron spectrometer during rendezvous, with quality of data improving over time

- Volatiles are elements and compounds with low boiling points, including nitrogen, water, carbon dioxide, hydrogen, and ammonia. Upon exposure to vacuum within the frost line, volatiles tend to sublime

- Neutron spectrometer likely able to measure about $1 \mathrm{~m}$ below surface of asteroid, depending on mean free path of neutrons through the body of the asteroid

- An unexpectedly high volatile composition under the surface affects momentum imparted onto the PHO by vaporizing upon exposure to vacuum and imparting its own momentum onto the object

- The exposure of a buried ice lens could impact momentum onto the PHO in an unexpected way. 


\section{Citations}

Asphaug, E., 2009, Growth and Evolution of Asteroids, Ann. Rev. Earth and PI. Sci. 37(1), pp. 413-448.

Parmentier, E.M., \& Head, J.W., 1981, Viscous Relaxation of Impact Craters on Icy Planetary Surfaces: Determination of Viscosity with Depth. Icarus, 47, pp. 100-111. 